\title{
Spectral asymptotics for magnetic Schrödinger operators in domains with corners
}

\author{
Ayman Kachmar and Abdallah Khochman
}

\begin{abstract}
This paper is on magnetic Schrodinger operators in two dimensional domains with corners. Semiclassical formulas are obtained for the sum and number of eigenvalues. The obtained results extend former formulas for smooth domains in [11] and [10] to piecewise smooth domains.
\end{abstract}

Mathematics Subject Classification (2010). 81Q10, 35J10, 35P15.

Keywords. Spectral Theory, Schrodinger Operator, semiclassical analysis, piecewise smooth domains.

\section{Introduction}

The spectral analysis of magnetic Schrödinger operators in domains with boundary has been the subject of many research papers in the last two decades. Apart from the mathematical interest behind the study of their spectra, magnetic Schrödinger operators in interior/exterior domains with various boundary conditions arise in several models of condensed matter physics, as superconductivity (see [15], [9], [16], and [17]), liquid crystals (see [13], and [19]), and Fermi gases (see [6]).

The present paper is devoted to the study of magnetic Schrödinger operators in domains with corners (piecewise smooth domains). The presence of corners in the domain has a strong effect on the spectrum of the operator. In particular, it is shown in [2] and [14] that the presence of corners decreases the value of the ground state energy of the operator compared with the case of smooth domains. Discussion of this effect in the framework of superconductivity is given in [3].

We give in a simple particular case, a brief presentation of the semiclassical results proved in this paper. Suppose for simplicity that $\Omega$ is a simply connected bounded domain in $\mathbb{R}^{2}$ and that $\mathbf{A}$ is a vector field such that $b=\operatorname{curl} \mathbf{A}$ is constant. Let $P_{h, \Omega}=-(h \nabla-i \mathbf{A})^{2}$ be the magnetic Laplacian in $L^{2}(\Omega)$ with magnetic Neumann boundary condition, $N(\lambda h)$ and $E(\lambda h)$ be the number and sum of negative eigenvalues of $P_{h, \Omega}-\lambda h I d$. If the boundary of $\Omega$ is smooth, it is proved in [10] and [11] that, 
as $h \rightarrow 0$,

$$
N(\lambda h) \sim c_{1}(b, \lambda)|\partial \Omega| h^{-1 / 2} \text { and } E(\lambda h) \sim c_{2}(b, \lambda)|\partial \Omega| h^{1 / 2},
$$

where $c_{1}$ and $c_{2}$ are two explicit constants. The formula for the number $N(\lambda h)$ is valid for all $\lambda<b$, while that for the energy $E(\lambda h)$ is valid for all $\lambda \leq b$.

In Theorem 4.1 of this paper, we prove that (1.1) is still holding true when the domain $\Omega$ has a finite number of corners. The key to prove this extension is a rough estimate on the number of eigenvalues of a Schrödinger operator with constant magnetic field in a sectorial domain (see Lemma 2.5).

The result of Theorem 4.1 shows that corners only affect the low-lying eigenvalues of the operator. As we will see in Theorem 3.1, corners create a few additional eigenvalues compared with smooth domains.

As a consequence, we may say that in the semi-classical limit and the regime considered in this paper, the numbers of eigenvalues of magnetic Schrödinger operators for smooth and non-smooth domains are asymptotically the same.

The paper is organized in the following way. In Section 2, we collect some key results that will be used throughout the paper. Section 3 is about the number of low-lying eigenvalues of the operator in a domain with corners (here the regularity assumption on the domain is precisely stated). Finally, Section 4 contains the semiclassical analysis that extends (1.1) to domains with corners.

Throughout the paper, the notation $N(\lambda, P)$ will be often used to denote the number of eigenvalues (counting multiplicities) of the operator $P$ that are below $\lambda$.

Acknowledgments. The authors are grateful for the anonymous referee for indicating many helpful suggestions and pointing out many corrections in the text of the paper. The research of the authors is funded by a grant from the Lebanese University.

\section{Preliminaries}

2.1. Variational principles. Let $H$ be a self-adjoint operator in a Hilbert space $\mathscr{H}$ (of domain $D(H)$ ) such that

$$
\left\{\begin{array}{l}
\inf \sigma_{\mathrm{ess}}(H) \geq 0 \\
H \mathbf{1}_{(-\infty, 0)}(H) \text { is trace class. }
\end{array}\right.
$$

We shall need the following two simple variational principles concerning the operator $H$, which are frequently used in [18] and [8].

Lemma 2.1. Let $\gamma$ be a bounded operator such that $0 \leq \gamma \leq 1$ (in the sense of quadratic forms) and the operator $\mathrm{H} \gamma$ is trace class. Then it holds that

$$
\operatorname{tr}\left(H \mathbf{1}_{(-\infty, 0)}(H)\right) \leq \operatorname{tr}(H \gamma)
$$


Lemma 2.2. Assume that the operator $H$ satisfies the hypothesis $(\mathrm{H})$. Then it holds that

$$
\operatorname{tr}\left(H \mathbf{1}_{(-\infty, 0)}(H)\right)=\inf \sum_{j=1}^{N}\left\langle f_{j}, H f_{j}\right\rangle,
$$

where the infimum is taken over all orthonormal families $\left\{f_{1}, f_{2}, \ldots, f_{N}\right\} \subset D(H)$ and $N \geq 1$.

2.2. A family of one-dimensional differential operators. Let us recall the main results obtained in [7] and [15] concerning the family of harmonic oscillators with Neumann boundary condition. Given $\xi \in \mathbb{R}$, we define the quadratic form

$$
B^{1}\left(\mathbb{R}_{+}\right) \ni u \mapsto q[\xi](u)=\int_{\mathbb{R}_{+}}\left|u^{\prime}(t)\right|^{2}+|(t-\xi) u(t)|^{2} d t,
$$

where, for a positive integer $k \in \mathbb{N}$ and a given interval $I \subseteq \mathbb{R}$, the space $B^{k}(I)$ is defined by

$$
B^{k}(I)=\left\{u \in H^{k}(I) ; t^{j} u(t) \in L^{2}(I), j=1, \ldots, k\right\} .
$$

Since the quadratic form (2.1) is closed and symmetric it defines a unique self-adjoint operator $\mathscr{L}[\xi]$. This operator has domain

$$
D(\mathscr{L}[\xi])=\left\{u \in B^{2}\left(\mathbb{R}_{+}\right) ; u^{\prime}(0)=0\right\},
$$

and is the realization of the differential operator

$$
\mathscr{L}[\xi]=-\partial_{t}^{2}+(t-\xi)^{2},
$$

on the given domain. We denote by $\left\{\mu_{j}(\xi)\right\}_{j=1}^{+\infty}$ the increasing sequence of eigenvalues of $\mathscr{L}[\xi]$, which are all simple. By the min-max principle, we have

$$
\mu_{1}(\xi)=\inf _{u \in B^{1}\left(\mathbb{R}_{+}\right), u \neq 0} \frac{q[\xi](u)}{\|u\|_{L^{2}\left(\mathbb{R}_{+}\right)}^{2}} .
$$

It follows from analytic perturbation theory (see [7]) that the functions

$$
\mathbb{R} \ni \xi \longmapsto \mu_{j}(\xi)
$$

are analytic. Furthermore, $\mu_{1}(0)=1, \mu_{1}(\xi) \leq 1$ for all $\xi \geq 0$, and $\left|\mu_{1}(\xi)-1\right|$ decays like $\exp \left(-\xi^{2}\right)$ as $\xi \rightarrow+\infty$ (see [4]). These properties of $\mu_{1}$ yield that

$$
\int_{0}^{\infty}\left(\mu_{1}(\xi)-1\right) d \xi=-\int_{\mathbb{R}}\left[\mu_{1}(\xi)-1\right]_{-} d \xi
$$

is finite. We define the constant

$$
\Theta_{0}=\inf _{\xi \in \mathbb{R}} \mu_{1}(\xi) .
$$


In [7], it is proved that $\Theta_{0}=\mu_{1}\left(\xi_{0}\right)$, that $\xi_{0}$ is the unique value at which the minimum $\Theta_{0}$ is attained and that $\mu^{\prime \prime}\left(\xi_{0}\right)>0$.

An important consequence of standard Sturm-Liouville theory is recalled below (c.f. Lemma 2.2 in [11]).

Lemma 2.3. The second eigenvalue of $\mathscr{L}[\xi]$ satisfies

$$
\inf _{\xi \in \mathbb{R}} \mu_{2}(\xi)>1 .
$$

Notice that part of this conclusion is a consequence of the analysis of Dauge and Helffer [7], who show that the infimum of $\mu_{2}(\xi)$ is attained for a unique $\xi_{2} \in \mathbb{R}$.

2.3. Rough energy bound for the cylinder. Let us consider the operator

$$
P_{h, \Omega}=-\left(h \nabla-i b \mathbf{A}_{0}\right)^{2} \quad \text { in } L^{2}(\Omega),
$$

with

$$
\Omega=[0, S] \times\left(0, h^{1 / 2} T\right) .
$$

Functions in the domain of $P_{h, \Omega}$ satisfy Neumann condition at $t=0$, periodic conditions at $s \in\{0, S\}$ and Dirichlet condition at $t=h^{1 / 2} T$. We assume that the vector field $\mathbf{A}_{0}$ is given by

$$
\mathbf{A}_{0}(s, t)=(-t, 0) .
$$

In this particular case, the operator has compact resolvent, hence the spectrum consists of an increasing sequence of eigenvalues $\left(e_{j}\right)_{j \geq 1}$ converging to $+\infty$. Note that the terms of the sequence $\left(e_{j}\right)$ are listed with multiplicities counted. Given $\lambda \in \mathbb{R}$, the energy

$$
\mathcal{E}(\lambda, b, S, T)=\sum_{j}\left[h b(1+\lambda)-e_{j}\right]_{+}
$$

is finite. The number of eigenvalues below $(1+\lambda) h b$ is also of particular interest:

$$
\mathcal{N}(\lambda, b, S, T)=\operatorname{Card}\left\{j: e_{j} \leq h b(1+\lambda)\right\} .
$$

A function in the domain of $P_{h, \Omega}$ is periodic with respect to the first variable ( $s$-variable) so that it can be expanded in a Fourier series. In the proof Lemma 2.4 below, expansion in a Fourier series is used to separate variables.

Lemma 2.4. There exist positive constants $T_{0}$ and $\lambda_{0}$ such that, for all $S>0, b>0$, $T \geq \sqrt{b} T_{0}$ and $\lambda \in\left(0, \lambda_{0}\right]$, we have

$$
\mathcal{E}(\lambda, b, S, T) \leq(1+\lambda) h b\left(\frac{S T}{2 \pi \sqrt{h}}+1\right)
$$

and

$$
\mathcal{N}(\lambda, b, S, T) \leq \frac{S T}{2 \pi \sqrt{h}}+1 .
$$


The lemma is proved in [10], although the estimate (2.9) is not stated explicitly. Actually, (2.8) is proved as follows. First (2.9) is established by separation of variables and the variational min-max principle then the energy $\varepsilon(\lambda, b, S, T)$ is easily estimated as $\mathcal{N}(\lambda, b, S, T) \times(1+\lambda) b h$, from which $(2.8)$ follows.

2.4. Rough bounds for the sector. Let $\alpha \in(0,2 \pi), R>0, h>0, b>0$ and

$$
\Omega_{R, h, \alpha}=\left\{(r \cos \theta, r \sin \theta) \in \mathbb{R}^{2}: 0<\theta<\alpha, 0 \leq r<h^{1 / 2} R\right\}
$$

Consider the self-adjoint operator $P_{h, \alpha}=-\left(h \nabla-i b \mathbf{A}_{0}\right)^{2}$ in $L^{2}\left(\Omega_{R, h, \alpha}\right)$. Functions in the domain of $P_{h, \alpha}$ satisfy Dirichlet condition on the boundary $r=h^{1 / 2} R$ and Neumann condition

$$
v \cdot\left(h \nabla-i b \mathbf{A}_{0}\right) u=0
$$

on the boundary defined by $\theta=0$ or $\theta=\alpha$. Here $v$ is the unit outward normal vector on the boundary $\partial \Omega_{R, h, \alpha}$ and $\mathbf{A}_{0}\left(x_{1}, x_{2}\right)=\left(-x_{2}, 0\right)$ is the magnetic potential introduced in (3.2).

The operator $P_{h, \alpha}$ has compact resolvent and its spectrum is discrete and consists of isolated eigenvalues $\left(e_{j}\right)$ counted with multiplicities. Let $\lambda \in \mathbb{R}$ and define

$$
\varepsilon_{\mathrm{corn}}(\alpha, b, R, \lambda)=\sum_{j}\left[h b(1+\lambda)-e_{j}\right]_{+},
$$

and

$$
\mathcal{N}_{\text {corn }}(\alpha, b, R, \lambda)=\operatorname{Card}\left\{j: e_{j} \leq h b(1+\lambda)\right\} .
$$

We give rough estimates of $\mathcal{E}_{\text {corn }}(\alpha, b, R, \lambda)$ and $\mathcal{N}_{\text {corn }}(\alpha, b, R, \lambda)$ in the next lemma.

Lemma 2.5. Given $b>0$ and $\alpha \in[0,2 \pi)$, there exist positive constants $C, h_{0}, R_{0}$ and $\lambda_{1}$ such that, for all $R \geq R_{0}, h \in\left(0, h_{0}\right]$, and $\lambda \in\left(-\infty, \lambda_{1}\right]$, we have

$$
\mathcal{N}_{\text {corn }}(\alpha, b, R, \lambda) \leq C\left(R^{2}+1\right),
$$

and

$$
\varepsilon_{\text {corn }}(\alpha, b, R, \lambda) \leq C(1+\lambda) h b\left(R^{2}+1\right) .
$$

Proof. Observe that the upper bound (2.14) on the energy $\mathcal{E}_{\mathrm{corn}}(\alpha, b, R, \lambda)$ follows immediately from the definition of $\mathcal{E}_{\mathrm{corn}}(\alpha, b, R, \lambda)$ and the upper bound in (2.13). Therefore, we establish the bound in (2.13). The method that will be used is introduced in [5] and based on a decomposition of the operator via a partition of unity and on the min-max variational principle.

To simplify the notation, we denote by $\Omega$ and $P$ the set $\Omega_{R, h, \alpha}$ introduced in (2.10) and the operator $P_{h, \alpha}$ respectively. 
Let $L_{0}$ be a positive real number whose choice will be specified later. Suppose $R>L_{0}$. We cover $\Omega$ by two sets

$$
U_{1}(h)=\left\{x \in \Omega:|x|<L_{0} h^{1 / 2}\right\},
$$

and

$$
U_{2}(h)=\left\{x \in \Omega: \frac{L_{0} h^{1 / 2}}{2}<|x|<R h^{1 / 2}\right\} .
$$

Consider a partition of unity of $\Omega$

$$
\sum_{j=1}^{2} \chi_{j}^{2}(x)=1 \quad \text { and } \quad \sum_{j=1}^{2}\left|\nabla \chi_{j}(x)\right|^{2} \leq \frac{C_{1}}{L_{0}^{2} h} \text { in } \Omega,
$$

where $C_{1}>0$ is a universal constant and $\operatorname{supp} \chi_{j} \subset U_{j}(h)$.

Let $u \in H^{1}(\Omega)$. The following decomposition formula holds:

$$
\int_{\Omega}\left|\left(h \nabla-i b \mathbf{A}_{0}\right) u\right|^{2} d x=\sum_{j=1}^{2} \int_{\Omega}\left(\left|\left(h \nabla-i b \mathbf{A}_{0}\right) \chi_{j} u\right|^{2}-h^{2}\left|\nabla \chi_{j}\right|^{2}|u|^{2}\right) d x .
$$

This decomposition yields the inequality stated below by using the upper bound on $\left|\nabla \chi_{j}\right|$ :

$$
\int_{\Omega}\left|\left(h \nabla-i b \mathbf{A}_{0}\right) u\right|^{2} d x \geq \sum_{j=1}^{2} \int_{\Omega}\left(\left|\left(h \nabla-i b \mathbf{A}_{0}\right) \chi_{j} u\right|^{2}-C_{1} L_{0}^{-2} h|u|^{2}\right) d x .
$$

By using the method in [5] and the variational min-max principle, we get that

$$
\mathcal{N}_{\text {corn }}(\alpha, b, R, \lambda) \leq N\left(\Lambda h, P_{U_{1}(h)}\right)+N\left(\Lambda h, P_{U_{2}(h)}\right),
$$

where $\Lambda=(1+\lambda) b+C_{1} L_{0}^{-2}, P_{U_{1}(h)}=-\left(h \nabla-i b \mathbf{A}_{0}\right)^{2}$ is the operator in $L^{2}\left(U_{1}(h)\right)$ with Dirichlet condition on $r=L_{0} h^{1 / 2}$ and Neumann condition on the other parts of the boundary of $U_{1}(h)$, and $P_{U_{2}(h)}=-\left(h \nabla-i b \mathbf{A}_{0}\right)^{2}$ is the operator in $L^{2}\left(U_{2}(h)\right)$ with Neumann boundary condition on $\theta=0$ and $\theta=\alpha$ and Dirichlet condition on the other parts of the boundary defined by $\theta=\alpha / 4, r=L_{0} h^{1 / 2} / 2$ and $r=R h^{1 / 2}$. Notice that we use polar coordinates $(r, \theta)$ in the definition of the domains.

We will need a technical assumption on the number $\lambda$. We try to explain the need of this technical assumption. While estimating the terms in the right side of (2.15), we will define the number

$$
\tilde{\lambda}=\lambda+2 b^{-1} C_{1} L_{0}^{-2}+C_{2} b^{-1} h .
$$

Recall the number of eigenvalues $\mathcal{N}\left(\tilde{\lambda}, b, R h^{1 / 2}, R\right)$ as introduced in (2.7). We shall see later that we need to estimate the number $\mathcal{N}\left(\tilde{\lambda}, b, R h^{1 / 2}, R\right)$. An estimate of this 
number is given in Lemma 2.4 provided that $\tilde{\lambda}$ is smaller than a certain constant $\lambda_{0}$ (this constant is introduced in Lemma 2.4). Suppose $\lambda_{1} \in\left(0, \lambda_{0}\right)$. If $h$ and $L_{0}^{-1}$ are selected sufficiently small compared with $\lambda_{1}$, it is possible to have $\tilde{\lambda}<\lambda_{0}$ whenever $\lambda \in\left[0, \lambda_{1}\right]$ (note that such an upper bound on $\tilde{\lambda}$ will fail if $\lambda$ is supposed to vary in the extended interval $\left.\left[0, \lambda_{0}\right]\right)$.

Suppose in the sequel that $\lambda \in\left[0, \lambda_{1}\right]$. We will prove that there exists a positive constant $C$ independently of $\lambda \in\left[0, \lambda_{1}\right]$ and such that

$$
N\left(\Lambda h, P_{U_{1}(h)}\right) \leq C
$$

and

$$
N\left(\Lambda h, P_{U_{2}(h)}\right) \leq C\left(R^{2}+1\right)
$$

In light of (2.15), Theorem 2.5 will be proved once the statements in (2.16) and (2.17) is shown to be true.

Proof of (2.16). The re-scaling $y=h^{-1 / 2} x$ gives immediately

$$
N\left(\Lambda h, P_{U_{1}(h)}\right)=N\left(\Lambda, P_{U_{1}(1)}\right)
$$

Here

$$
P_{U_{1}(1)}=-\left(\nabla-i b \mathbf{A}_{0}\right)^{2} \quad \text { in } L^{2}\left(U_{1}(1)\right) \text {. }
$$

Since the operator $P_{U_{1}(1)}$ has compact resolvent and the number $\Lambda$ is bounded independently of $h \in(0,1]$, it follows that $N\left(\Lambda, P_{U_{1}(1)}\right)$ is bounded as $h$ varies in $(0,1]$ too. This establishes the upper bound in $(2.16)$.

Proof of (2.17). The proof requires introducing an additional parameter $\varepsilon$ as follows:

$$
0<\varepsilon<\min \left(\frac{\alpha}{8}, \frac{\pi}{4}-\frac{\alpha}{8}\right)
$$

The need for this parameter is rather technical. Let us explain the idea that will be used. The domain $U_{2}(h)$ will be covered by four sectorial domains with openings not exceeding $\alpha / 4+2 \varepsilon$ and overlapping in a region with area proportional to $\varepsilon$. With the choice of $\varepsilon$, we see that the openings of the sectorial domains do not exceed $\pi / 2$ and as a consequence, each of these domains is included inside a rectangle. In a rectangular domain, it is possible to estimate the number of eigenvalues of Schrödinger operators as in Lemma 2.4. Details will follow below.

Recall the definition of the set

$$
U_{2}(h)=\left\{x \in \Omega: \frac{L_{0} h^{1 / 2}}{2}<|x|<R h^{1 / 2}\right\} .
$$


We cover $U_{2}(h)$ by four sets

$$
\begin{aligned}
& V_{1}(h)=\left\{(r \cos \theta, r \sin \theta) \in \overline{U_{2}(h)}: 0 \leq \theta<\frac{\alpha}{4}\right\}, \\
& V_{2}(h)=\left\{(r \cos \theta, r \sin \theta) \in \overline{U_{2}(h)}: \frac{\alpha}{4}-\varepsilon<\theta<\frac{\alpha}{2}+\varepsilon\right\}, \\
& V_{3}(h)=\left\{(r \cos \theta, r \sin \theta) \in \overline{U_{2}(h)}: \frac{\alpha}{2}-\varepsilon<\theta<\frac{3 \alpha}{4}+\varepsilon\right\}
\end{aligned}
$$

and

$$
V_{4}(h)=\left\{(r \cos \theta, r \sin \theta) \in \overline{U_{2}(h)}: \frac{3 \alpha}{4}<\theta \leq \alpha\right\} .
$$

Define four operators $P_{V_{1}(h)}, P_{V_{2}(h)}, P_{V_{3}(h)}$, and $P_{V_{4}(h)}$ in $L^{2}\left(V_{1}(h)\right), L^{2}\left(V_{2}(h)\right)$, $L^{2}\left(V_{3}(h)\right)$, and $L^{2}\left(V_{4}(h)\right)$ respectively. The four operators are self-adjoint realizations of the differential operator $-\left(h \nabla-i b \mathbf{A}_{0}\right)^{2}$. Functions in the domain of $P_{V_{1}(h)}$ satisfy Neumann condition on $\theta=0$ and Dirichlet condition on the other parts of the boundary defined by $r=L_{0} h^{1 / 2} / 2$ and $r=L_{0} h^{1 / 2}$. Similarly, functions in the domain of $P_{V_{4}(h)}$ satisfy Neumann condition on $\theta=\alpha$ and Dirichlet condition on the other parts of the boundary. Functions in the domains of $P_{V_{2}(h)}$ and $P_{V_{3}(h)}$ satisfy Dirichelt boundary condition.

Notice that the operators $P_{V_{1}(h)}$ and $P_{V_{4}(h)}$ are unitary equivalent and hence have same spectra. Also, the operators $P_{V_{2}(h)}$ and $P_{V_{3}(h)}$ are unitary equivalent and have same spectra.

We apply an argument similar to the one we did to obtain (2.15). By introducing a partition of unity supported in $V_{1}(h), V_{2}(h), V_{3}(h)$ and, $V_{4}(h)$, using the IMS decomposition formula and the variational min-max principle, we get a constant $C_{2}>0$ that is allowed to depend on $\varepsilon$ but not on $h$ and such that

$$
\begin{aligned}
N\left(\Lambda h, P_{U_{2}(h)}\right) & \leq \sum_{j=1}^{4} N\left(\tilde{\Lambda} h, P_{V_{j}(h)}\right) \\
& =2 \sum_{j=1}^{2} N\left(\tilde{\Lambda} h, P_{V_{j}(h)}\right),
\end{aligned}
$$

with $\widetilde{\Lambda}=\Lambda+C_{1} L_{0}^{-2}+C_{2} h$.

Recall the number $\mathcal{N}(\lambda, b, S, T)$ introduced in (2.7). This number counts the eigenvalues of the operator $P_{h, U_{S, T}}$ in the cylinder $U_{S, T}=[0, S] \times\left(0, h^{1 / 2} T\right)$.

Since the angle $\alpha / 4$ is acute and $R>L_{0}$, we see that

$$
V_{1}(h) \subset\left[0, R h^{1 / 2}\right] \times\left(0, R h^{1 / 2}\right) .
$$

A function $u\left(x_{1}, x_{2}\right)$ in the form domain of $P_{V_{1}(h)}$ satisfies Dirichlet boundary condition on

$$
r=L_{0} h^{1 / 2} / 2, \quad r=L_{0} h^{1 / 2}, \quad \text { and } \quad \theta=\alpha / 4 .
$$


As a consequence, such a function $u\left(x_{1}, x_{2}\right)$ in the form domain of $P_{V_{1}(h)}$ can be extended by zero to a function $\tilde{u}\left(x_{1}, x_{2}\right)$ defined in the cylinder

$$
U \stackrel{\text { def }}{=}\left[0, R h^{1 / 2}\right] \times\left(0, R h^{1 / 2}\right) .
$$

The extended function $\tilde{u}\left(x_{1}, x_{2}\right)$ satisfies Dirichlet condition on $x_{1}=0, x_{1}=R h^{1 / 2}$ and $x_{2}=R h^{1 / 2}$. In particular $\tilde{u}\left(x_{1}, x_{2}\right)$ is periodic with respect to $x_{1}$ and is inside the form domain of $P_{h, U}$. In this way, we see that the form domain of the operator $P_{V_{1}(h)}$ is embedded in the form domain of the operator $P_{h, U}$.

Thus, by the variational min-max principle, it is easy to see that

$$
N\left(\tilde{\Lambda} h, P_{V_{1}(h)}\right) \leq \mathcal{N}\left(\tilde{\lambda}, b, R h^{1 / 2}, R\right),
$$

with $\tilde{\lambda}=\lambda+2 b^{-1} C_{1} L_{0}^{-2}+C_{2} b^{-1} h$ (remember that $\tilde{\Lambda}=\Lambda+C_{1} L_{0}^{-2}+C_{2} h$, and $\mathcal{N}\left(\tilde{\lambda}, b, R h^{1 / 2}, R\right)$ counts the eigenvalues $\left.\leq(1+\tilde{\lambda}) b h\right)$.

Similarly, $V_{2}(h)$ is inside a rectangular domain $D$. Rotating the rectangular domain $D$ transforms it to the domain $U$ introduced above. Notice that the action of a rotation is a unitary transformation on the operator $P_{V_{2}(h)}$ and does not change the spectrum. In this way, functions in the form domain of $P_{V_{2}(h)}$ are embedded in the form domain of $P_{h, U}$ via unitary transformations (rotation of the variable and extension by 0 ). The min-max principle will also give us that

$$
N\left(\tilde{\Lambda} h, P_{V_{2}(h)}\right) \leq \mathcal{N}\left(\tilde{\lambda}, b, R h^{1 / 2}, R\right) .
$$

As a consequence, we infer from (2.18)

$$
N\left(\Lambda h, P_{U_{2}(h)}\right) \leq 4 \mathcal{N}\left(\tilde{\lambda}, b, R h^{1 / 2}, R\right) .
$$

Select $h_{0}$ sufficiently small and $L_{0}$ sufficiently large such that

$$
2 b^{-1} C_{1} L_{0}^{-2}+C_{2} b^{-1} h_{0}<\lambda_{0}-\lambda_{1} .
$$

In this way, we get for all $\lambda \in\left(0, \lambda_{1}\right)$ and $h \in\left(0, h_{0}\right]$

$$
\tilde{\lambda}=\lambda+2 b^{-1} C_{1} L_{0}^{-2}+C_{2} b^{-1} h_{0} \in\left(0, \lambda_{0}\right] .
$$

Consequently, it follows from Lemma 2.4 that $\mathcal{N}\left(\tilde{\lambda}, b, R h^{1 / 2}, R\right)$ is bounded by a constant times $\left(R^{2}+1\right)$, and thereby get the upper bound in (2.17).

\section{Asymptotic number of low-lying eigenvalues near corners}

3.1. The operator in an infinite sector. Let $\alpha \in(0,2 \pi)$ and

$$
\Omega_{\alpha}=\left\{(r \cos \theta, r \sin \theta) \in \mathbb{R}^{2}: r \geq 0,0<\theta<\alpha\right\} .
$$


Define the magnetic potential

$$
\mathbf{A}_{0}\left(x_{1}, x_{2}\right)=\left(-x_{2}, 0\right), \quad\left(x_{1}, x_{2}\right) \in \mathbb{R}^{2},
$$

whose curl is constant and equal to 1 .

Consider the self-adjoint operator $P_{\Omega_{\alpha}}=-\left(\nabla-i \mathbf{A}_{0}\right)^{2}$ in $L^{2}\left(\Omega_{\alpha}\right)$ whose domain is

$$
\begin{aligned}
& D\left(P_{\Omega_{\alpha}}\right)=\left\{u \in L^{2}\left(\Omega_{\alpha}\right):\right.\left(\nabla-i \mathbf{A}_{0}\right) u \in L^{2}(\Omega), P_{\Omega_{\alpha}} u \in L^{2}\left(\Omega_{\alpha}\right), \\
&\left.v \cdot\left(\nabla-i \mathbf{A}_{0}\right) u=0 \text { on } \partial \Omega_{\alpha} \backslash\{0\}\right\},
\end{aligned}
$$

where $v$ is the unit outward normal vector of $\partial \Omega_{\alpha}$.

It is proved in [2] that the bottom of the essential spectrum of $P_{\Omega_{\alpha}}$ is a universal constant $\Theta_{0} \in\left(\frac{1}{2}, 1\right)$. Furthermore, when $\alpha \in\left(0, \frac{\pi}{2}\right]$, the operator $P_{\Omega_{\alpha}}$ has discrete spectrum below $\Theta_{0}$.

Many questions connected with the spectrum of the operator $P_{\Omega_{\alpha}}$ are left open. Among these questions are the following onces.

- Is the spectrum of $P_{\Omega_{\alpha}}$ below $\Theta_{0}$ finite?

- When $\alpha=\pi$, the operator $P_{\Omega_{\pi}}$ is the half-plane and its spectrum is purely essential and consists of the interval $\left[\Theta_{0}, \infty\right)$. Is $\left[\Theta_{0}, \infty\right)$ the essential spectrum of $P_{\Omega_{\alpha}}$ for any $\alpha$ ?

- It is conjectured in [2] that the ground state energy (bottom of the spectrum) of $P_{\Omega_{\alpha}}$ is constant and equal to $\Theta_{0}$ when $\alpha \in[\pi, 2 \pi)$. This conjecture has not been proved or disproved yet (see [2]).

3.2. Assumptions on the domain. We describe precisely the regularity properties of the domain $\Omega$. The assumptions will be the same as those made in [2] and [3].

In this and the subsequent sections, $\Omega$ is an open and connected set in $\mathbb{R}^{2}$ whose boundary is compact and consists of a curvilinear polygon of class $C^{3}$. By saying that the boundary $\Gamma$ of $\Omega$ is a curvilinear polygon (of class $C^{3}$ ) we mean the following (see [12], p. 34-42). For every $x \in \Gamma$, there exists a neighborhood $V$ of $x$ in $\mathbb{R}^{2}$ and a mapping $\psi$ from $V$ to $\mathbb{R}^{2}$ such that

(1) $\psi$ is injective,

(2) $\psi$ together with $\psi^{-1}$ (defined on $\psi(V)$ ) belongs to the class $C^{3}$,

(3) $\Omega \cap V$ is either $\left\{y \in \Omega: \psi_{2}(y)<0\right\},\left\{y \in \Omega: \psi_{1}(y)<0\right.$ and $\left.\psi_{2}(y)<0\right\}$, or $\left\{y \in \Omega: \psi_{1}(y)<0\right.$ or $\left.\psi_{2}(y)<0\right\}$, where $\psi_{j}$ denotes the components of $\psi$.

The boundary $\Gamma$ of $\Omega$ is a piecewise smooth curve. We work under the assumption that the boundary $\Gamma$ consists of a finite number of smooth curves $\bar{\Gamma}_{k}$ for $k=1, \ldots, m$. The family $\left(\Gamma_{k}\right)$ is the minimal family of curves making up the boundary $\Gamma$. If $\Gamma$ consists of more than one smooth curve $(m \geq 2)$, then we suppose that the curve $\bar{\Gamma}_{k+1}$ follows $\bar{\Gamma}_{k}$ according to a positive orientation, on each connected component 
of $\Gamma$. Let $s_{k}$ denotes the vertex which is the end point of $\bar{\Gamma}_{k}$. In a neighborhood of $\partial \Omega$, define a vector field $v_{k}$. For each $k$, the vector $v_{k}$ is the unit normal a.e. on $\Gamma_{k}$.

Let $\Sigma$ be the set of vertices the domain $\Omega$ has. We suppose that $\Sigma \neq \emptyset$. Under this assumption $m \geq 2$ and $\Sigma$ consists exactly of $m$ vertices. This assumption certifies that the domain $\Omega$ does not have a smooth boundary but only a piecewise smooth boundary. When $\Sigma=\emptyset$, then $m=1$, the boundary of $\Omega$ is smooth and the operator in $L^{2}(\Omega)$ is the one studied in [11] and [10].

At each vertex $\mathrm{s}_{k} \in \Sigma$, let $\alpha_{\mathrm{s}_{\mathrm{k}}}$ denotes the angle between $\bar{\Gamma}_{k}$ and $\bar{\Gamma}_{k+1}$ measured towards the interior.

3.3. Main result. For each angle $\alpha$, recall the sectorial domain $\Omega_{\alpha}$ introduced in (3.1). Let $P_{\mathfrak{b}, \Omega_{\alpha}}=-\left(\nabla-i \mathfrak{b} \mathbf{A}_{0}\right)^{2}$ be the operator in $L^{2}\left(\Omega_{\alpha}\right)$ introduced in Section 3.1 (with Neumann boundary condition on the smooth part of the boundary of the sector $\Omega_{\alpha}$ ). The number

$$
\mathrm{n}(\alpha, \lambda ; \mathfrak{b})=\operatorname{tr}\left(\mathbf{1}_{(-\infty, \lambda)}\left(P_{\mathfrak{b}, \Omega_{\alpha}}\right)\right)=\sum_{\Lambda<\lambda} \operatorname{dim}\left(\operatorname{Ker}\left(P_{\mathfrak{b}, \Omega_{\alpha}}-\Lambda \mathrm{Id}\right)\right)
$$

is finite for all $\alpha$ and $\lambda<\Theta_{0}$.

If $\mathfrak{b}=1$, we write

$$
\mathrm{n}(\alpha, \lambda)=\mathrm{n}(\alpha, \lambda ; \mathfrak{b}=1) .
$$

By a scaling argument, it is easy to see that

$$
\mathrm{n}(\alpha, \lambda ; \mathfrak{b})=\mathrm{n}(\alpha, \lambda / \mathfrak{b})
$$

Let the domain $\Omega$ be as described in Section 3.2. Define the magnetic Schrödinger operator

$$
P_{h, \Omega}=-(h \nabla-i \mathbf{A})^{2}, \quad \text { in } L^{2}(\Omega),
$$

where $\mathbf{A} \in C^{2}\left(\Omega ; \mathbb{R}^{2}\right)$ is the magnetic potential, $h>0$ is the semi-classical parameter and $\mathbf{B}=\operatorname{curl} \mathbf{A}$ is the magnetic field. The domain of the operator $P_{h, \Omega}$ is

$$
\begin{aligned}
& D\left(P_{h, \Omega}\right)=\left\{u \in L^{2}(\Omega):\right.(h \nabla-i \mathbf{A})^{k} \in L^{2}(\Omega), k=1,2, \\
&\left.v_{j} \cdot(h \nabla-i \mathbf{A}) u=0 \text { on } \Gamma_{k}, k=1, \ldots, m\right\} .
\end{aligned}
$$

Define two constants

$$
b=\inf _{x \in \bar{\Omega}} \mathbf{B}(x) \quad \text { and } \quad b^{\prime}=\inf _{x \in \partial \Omega} \mathbf{B}(x) .
$$

The main result of this section is the following theorem.

Theorem 3.1. Let the constant $\Theta_{0}$ be as defined in (2.5), $\lambda \in\left(-\infty, \min \left(\Theta_{0} b^{\prime}, b\right)\right)$ and $N(\lambda h)$ the number of eigenvalues of the operator $P_{h, \Omega}$ below $\lambda$ h counting multiplicities. Suppose that the magnetic field $\mathbf{B}(x)$ is selected such that $b>0$. There 
exists a positive number $h_{0}$ such that, for all $h \in\left(0, h_{0}\right)$, the following equality holds,

$$
N(\lambda h)=\sum_{k=1}^{m} \mathrm{n}\left(\alpha_{\mathrm{s}_{k}}, \frac{\lambda}{\mathbf{B}\left(\mathrm{s}_{k}\right)}\right) .
$$

The number $h_{0}$ depends only on the angles $\alpha_{\mathrm{s}_{k}}$ and the domain $\Omega$.

Theorem 3.1 gives the exact number of low-lying eigenvalues of $P_{h, \Omega}$ corresponding to corners in the domain $\Omega$. When $\lambda>\Theta_{0}$, we will see in Section 4 that corners no more affect the leading order term of $N(\lambda h)$.

\subsection{Proof of Theorem 3.1.}

Upper bound. Let $\rho$ be a positive constant satisfying $0<\rho<1$. We start by choosing a partition of unity $\chi_{k, h}$ introduced in Proposition 11.2 in [2] satisfying

$$
\sum_{k}\left|\chi_{k, h}\right|^{2}=1, \quad \sum_{k}\left|\nabla \chi_{k, h}\right|^{2} \leq C h^{-2 \rho} \quad \text { in } \mathbb{R}^{2}
$$

and

$$
\operatorname{supp} \chi_{k, h} \subset B\left(z_{j}, c_{k} h^{\rho}\right),
$$

with the choice of indices such that

- $z_{k}=\mathrm{s}_{k}$ and $c_{k}=1$ for all $k=1,2, \ldots, m$;

- if $k \notin\{1, \ldots, m\}$ and $z_{k} \notin \partial \Omega$, then

$$
B\left(z_{k}, c_{k} h^{\rho}\right) \cap \partial \Omega=\emptyset
$$

and

$$
c_{k}=\frac{1}{2} \min \left(\left|\tan \alpha_{s_{k}}\right|, 1\right)
$$

- if $k \notin\{1, \ldots, m\}$ and $z_{k} \in \partial \Omega$, then

$$
\begin{aligned}
& B\left(z_{k}, c_{k} h^{\rho}\right) \cap \Sigma=\emptyset, \\
& B\left(z_{k}, c_{k} h^{\rho}\right) \cap \partial \Omega \text { is connected, }
\end{aligned}
$$

and

$$
c_{k}=\frac{1}{2} \min \left(\left|\tan \alpha_{\mathrm{s}_{k}}\right|, 1\right) .
$$


Recall that $\Sigma$ is the set of vertices of the domain $\Omega$. If $u$ is a function in the form domain of $P_{h, \Omega}$, define

$$
q(u)=\int_{\Omega}|(h \nabla-i \mathbf{A}) u|^{2} d x .
$$

The following decomposition formula holds true for every function $u$ in the form domain of the operator $P_{h, \Omega}$ :

$$
\begin{aligned}
q(u)= & \sum_{k=1}^{m} q\left(\chi_{k, h} u\right)+\sum_{\substack{k>m \\
z_{k} \in \partial \Omega}} q\left(\chi_{k, h} u\right) \\
& +\sum_{\substack{k>m \\
z_{k} \notin \partial \Omega}} q\left(\chi_{k, h} u\right)-h^{2} \sum_{k} \int_{\Omega}\left|\nabla \chi_{k, h}\right|^{2}|u|^{2} d x .
\end{aligned}
$$

Using the upper bound on $\left|\nabla \chi_{k, h}\right|^{2}$, we get the lower bound

$$
\begin{aligned}
q(u) \geq & \sum_{k=1}^{m} q\left(\chi_{k, h} u\right)+\sum_{\substack{k>m \\
z_{k} \in \partial \Omega}} q\left(\chi_{k, h} u\right) \\
& +\sum_{\substack{k>m \\
z_{k} \notin \partial \Omega}} q\left(\chi_{k, h} u\right)-C h^{2-2 \rho} \int_{\Omega}|u|^{2} d x .
\end{aligned}
$$

Let $k \in\{1,2, \ldots, m\}$. It is proved in [2], p. 252, that by performing a change of variable $y=\psi_{k}(x)$ and a gauge transformation (defined by a function $\varphi_{k}$ ), the following lower bound holds true:

$$
q\left(\chi_{k, h} u\right) \geq \int_{\Omega_{\alpha_{s_{k}}}}\left(\left(1-C h^{\rho}-C h^{2 \theta}\right)\left|\left(h \nabla-i B_{k} \mathbf{A}_{0}\right) v_{k}\right|^{2}-C h^{4 \rho-2 \theta}\left|v_{k}\right|^{2}\right) d y .
$$

Here $\theta \in(0,1)$ is any constant, $B_{k}=\mathbf{B}\left(z_{k}\right), \mathbf{A}_{0}$ is the magnetic potential in (3.2) and

$$
v_{k}(y)=e^{-i \varphi_{k}(y)}\left(\chi_{k, h} u\right) \circ \psi_{k}^{-1}(y) .
$$

The optimal choice of $\rho$ and $\theta$ is $\rho=3 / 8$ and $\theta=1 / 8$. This produces the lower bound

$$
q\left(\chi_{k, h} u\right) \geq \int_{\Omega_{\alpha_{s_{k}}}}\left(\left(1-C h^{1 / 4}\right)\left|\left(h \nabla-i B_{k} \mathbf{A}_{0}\right) v_{k}\right|^{2}-C h^{5 / 4}\left|v_{k}\right|^{2}\right) d y .
$$

Similarly, when $k \notin\{1,2, \ldots, m\}$ and $z_{k} \in \partial \Omega$, by applying a change of variable and a gauge transformation, we obtain the lower bound (see [11])

$$
q\left(\chi_{k, h} u\right) \geq \int_{U_{h}}\left(\left(1-C h^{1 / 4}\right)\left|\left(h \nabla-i B_{k} \mathbf{A}_{0}\right) v_{k}\right|^{2}-C h^{5 / 4}\left|v_{k}\right|^{2}\right) d y,
$$


where $U_{h}=\left\{y=\left(y_{1}, y_{2}\right) \in \mathbb{R}^{2}: y_{1} \in\left(0, h^{3 / 8}\right)\right.$ and $\left.y_{2} \in(0, \infty)\right\}$. By inserting the lower bounds in (3.11) and (3.12) into (3.9) we obtain

$$
\begin{aligned}
q(u) \geq & \sum_{k=1}^{m} \int_{\Omega_{\alpha_{s_{k}}}}\left(\left(1-C h^{1 / 4}\right)\left|\left(h \nabla-i B_{k} \mathbf{A}_{0}\right) v_{k}\right|^{2}-C h^{5 / 4}\left|v_{k}\right|^{2}\right) d y \\
& +\sum_{\substack{k>m \\
z_{k} \in \partial \Omega}} \int_{U_{h}}\left(\left(1-C h^{1 / 4}\right)\left|\left(h \nabla-i B_{k} \mathbf{A}_{0}\right) v_{k}\right|^{2}-C h^{5 / 4}\left|v_{k}\right|^{2}\right) d y \\
& +\sum_{\substack{k>m \\
z_{k} \notin \partial \Omega}} q\left(\chi_{k, h} u\right)-C h^{5 / 4} \int_{\Omega}|u|^{2} d x .
\end{aligned}
$$

By the variational min-max principle (and the remark in (3.5)), we deduce the following upper bound on the number $N(\lambda h)$ :

$$
\begin{aligned}
N(\lambda h) \leq & \sum_{k=1}^{m} \mathrm{n}\left(\alpha_{\mathrm{s}_{k}}, \frac{\lambda+C h^{1 / 4}}{\left(1-C h^{1 / 4}\right) \mathbf{B}\left(\mathrm{s}_{k}\right)}\right) \\
& +\sum_{\substack{k>m \\
z_{k} \in \partial \Omega}} N\left(P_{B_{k}, U_{h}}, \frac{\lambda h+C h^{5 / 4}}{1-C h^{1 / 4}}\right) \\
& +\sum_{\substack{k>m \\
z_{k} \notin \partial \Omega \Omega}} N\left(P_{h, \Omega}^{D}, \lambda h+C h^{5 / 4}\right) .
\end{aligned}
$$

Here $P_{B_{k}, U_{h}}=-\left(h \nabla-i B_{k} \mathbf{A}_{0}\right)^{2}$ is the operator in $L^{2}\left(U_{h}\right)$ with Neumann boundary condition at $y_{2}=0$ and Dirichlet condition elsewhere, and $P_{h, \Omega}^{D}=-(h \nabla-i \mathbf{A})^{2}$ is the operator in $L^{2}(\Omega)$ with Dirichlet boundary condition. The spectrum of $P_{B_{k}, U_{h}}$ starts at $\Theta_{0} B_{k} h$ and that of $P_{h, \Omega}^{D}$ starts above $b h$ (cf. Section 2 in [15] or Lemma 3.2 and eq. (1.4) in [11]). Thus, if $\lambda<\min \left(\Theta_{0} b^{\prime}, b\right)$ and $h$ is selected sufficiently small, we get

$$
N\left(P_{B_{k}, U_{h}}, \frac{\lambda h+C h^{5 / 4}}{1-C h^{1 / 4}}\right)=N\left(P_{h, \Omega}^{D}, \lambda h+C h^{5 / 4}\right)=0 .
$$

Since the spectrum of the operator $P_{\Omega_{\alpha_{s_{k}}}}$ below $\Theta_{0}$ consists of isolated eigenvalues, we get for $h$ sufficiently small that

$$
\mathrm{n}\left(\alpha_{\mathrm{s}_{k}}, \frac{\lambda+C h^{1 / 4}}{\left(1-C h^{1 / 4}\right) \mathbf{B}\left(\mathrm{s}_{k}\right)}\right)=\mathrm{n}\left(\alpha_{\mathrm{s}_{k}}, \frac{\lambda}{\mathbf{B}\left(\mathrm{s}_{k}\right)}\right) .
$$

This finishes the proof of the upper bound. 
Lower bound. The proof of the lower bound is similar to the one in [11] and uses a bracketing technique. Let $\widetilde{P}_{h, \Omega}$ be the self adjoint realization of the restriction of the operator $P_{h, \Omega}$ on functions vanishing outside the set

$$
\bigcup_{k=1}^{N} B\left(\mathrm{~s}_{k}, h^{3 / 8}\right)
$$

By the variational min-max principle, the eigenvalues of $\widetilde{P}_{h, \Omega}$ are larger than those of $P_{h, \Omega}$. Thus

$$
N(\lambda h) \geq N\left(\lambda h, \widetilde{P}_{h, \Omega}\right)
$$

We will show next that there exist constants $C>0$ and $h_{0}>0$ such that, for all $h \in\left(0, h_{0}\right]$, we have

$$
N\left(\lambda h, \widetilde{P}_{h, \Omega}\right) \geq \sum_{k=1}^{N} \mathrm{n}\left(\alpha_{\mathrm{s}_{k}}, \frac{\lambda-C h^{1 / 4}}{\left(1+C h^{1 / 4}\right) \mathbf{B}\left(\mathrm{s}_{k}\right)}\right)
$$

If $h$ is made sufficiently small, then

$$
\mathrm{n}\left(\alpha_{\mathrm{s}_{k}}, \frac{\lambda-C h^{1 / 4}}{\left(1+C h^{1 / 4}\right) \mathbf{B}\left(\mathrm{s}_{k}\right)}\right)=\mathrm{n}\left(\alpha_{\mathrm{s}_{k}}, \frac{\lambda}{\mathbf{B}\left(\mathrm{s}_{k}\right)}\right)
$$

and we get the lower bound we wish to prove.

Derivation of (3.14) is easy. Let $\tilde{q}$ be the quadratic form associated with $\widetilde{P}_{h, \Omega}$.

Select an arbitrary function $u$ in the form domain of $\widetilde{P}_{h, \Omega}$. A matching asymptotic upper bound of (3.11) is proved in [2], p. 252:

$$
\begin{aligned}
& \int_{B\left(\mathrm{~s}_{k}, h^{3 / 8}\right)}|(h \nabla-i \mathbf{A}) u|^{2} d x \\
& \quad \leq \int_{\Omega_{\alpha_{s_{k}}}}\left(\left(1-C h^{1 / 4}\right)\left|\left(h \nabla-i B_{k} \mathbf{A}_{0}\right) v_{j}\right|^{2}-C h^{5 / 4}\left|v_{k}\right|^{2}\right) d y,
\end{aligned}
$$

where $\mathbf{B}_{k}=\mathbf{B}\left(\mathrm{s}_{k}\right)$ and $v_{k}$ is obtained from $u$ by the change of variables $y=\psi_{k}(x)$ times a gauge transformation. Summing over $k$, we get

$$
\tilde{q}(u) \leq \sum_{k} \int_{\Omega_{\alpha_{\mathrm{s}_{k}}}}\left(\left(1-C h^{1 / 4}\right)\left|\left(h \nabla-i B_{k} \mathbf{A}_{0}\right) v_{k}\right|^{2}-C h^{5 / 4}\left|v_{k}\right|^{2}\right) d y .
$$

By the variational min-max principle, we deduce that the eigenvalues of the operator $\widetilde{P}_{h, \Omega}$ are smaller than those of the direct sum of the operators $P_{\Omega_{\alpha_{s}}, \mathbf{B}_{k}}$, thereby proving (3.14). 


\section{Energy and number of eigenvalues in piecewise smooth domains}

4.1. Main results. We will state other results concerning the number and sum of eigenvalues of the operator $P_{h, \Omega}$ introduced in (3.6). The assumptions on the domain $\Omega$ is as described in Section 3.2. The notation and assumption on the magnetic field $\mathbf{B}=\operatorname{curl} \mathbf{A}$ is as in (3.7) and Section 3.4.

Let $\left(e_{j}\right)$ be the increasing sequence of eigenvalues of the operator $P_{h, \Omega}$ in the interval $(-\infty, b h]$, counting multiplicities. If $\lambda \in(-\infty, b]$, define

$$
N(\lambda h)=\operatorname{tr}\left(\mathbf{1}_{(-\infty, \lambda h)}\left(P_{h, \Omega}\right)\right)=\operatorname{Card}\left\{e_{j}: e_{j} \leq \lambda h\right\}
$$

and

$$
E(\lambda h)=\operatorname{tr}\left(\mathbf{1}_{(-\infty, \lambda h)}\left(P_{h, \Omega}-\lambda h \mathrm{Id}\right)\right)=\sum_{e_{j} \leq \lambda h}\left(e_{j}-\lambda h\right)
$$

The main result we prove in this section is Theorem 4.1. Its statement requires the notation $[x]_{+}=\max (x, 0)$ for any real number $x$, the eigenvalue $\mu_{1}(\xi)$ introduced in (2.4), and the arc-length measure $d s(x)$ along the boundary of $\Omega$.

Theorem 4.1. For any real number $\lambda \leq b$ and as $h \rightarrow 0$, it holds true that

$$
E(\lambda h)=-\frac{h^{1 / 2}}{2 \pi} \int_{\partial \Omega} \int_{-\infty}^{\infty} \mathbf{B}(x)^{3 / 2}\left[\frac{\lambda}{\mathbf{B}(x)}-\mu_{1}(\xi)\right]_{+} d \xi d s(x)+o\left(h^{1 / 2}\right) .
$$

Furthermore, if $\lambda<b$, then

$$
N(\lambda h)=\frac{1}{2 \pi h^{1 / 2}} \int_{\left\{(x, \xi) \in \partial \Omega \times \mathbb{R}: \mathbf{B}(x) \mu_{1}(\xi) \leq \lambda\right.} \mathbf{B}(x)^{1 / 2} d \xi d s(x)+o\left(h^{-1 / 2}\right) .
$$

While proving Theorem 4.1, we only give the new ingredients and constructions required to adapt the proof given in [10]. We will refer to [10] for the detailed calculations.

4.2. Proof of Theorem 4.1. Notice that (4.4) is a consequence of (4.3). Actually, the term on the right side of (4.4) is the derivative with respect to $\lambda h$ of that on the right side of (4.3). On the other hand, using the variational principle of Lemma 2.2, the number $N(\lambda h)$ can be seen as the derivative of the energy $E(\lambda h)$ with respect to $\lambda h$.

Therefore, to prove Theorem 4.1, it is sufficient to establish the asymptotic formula in (4.3).

Using a magnetic Lieb-Thirring inequality and a decomposition of the operator by a partition of unity, it is possible to prove that the energy $E(\lambda h)$ is finite for all $\lambda \leq b$. Details regarding this proof are given in Section 5.1 in [10], pp. 242-243. 
We start by proving the asymptotic lower bound

$$
E(\lambda h) \geq-\frac{h^{1 / 2}}{2 \pi} \int_{\partial \Omega} \int_{-\infty}^{\infty} \mathbf{B}(x)^{3 / 2}\left[\frac{\lambda}{\mathbf{B}(x)}-\mu_{1}(\xi)\right]_{+} d \xi d s(x)+o\left(h^{1 / 2}\right) .
$$

Let $H=P_{h, \Omega}-\lambda h \mathrm{Id}$ and notice that the energy $E(\lambda h)$ can be expressed in the more useful form

$$
E(\lambda h)=\operatorname{tr}\left(H \mathbf{1}_{(-\infty, 0)}(H)\right) .
$$

Let $\left\{f_{1}, f_{2}, \ldots, f_{N}\right\}$ be any $L^{2}$ orthonormal set in $D(H)$. We will give a uniform lower bound of

$$
\sum_{j=1}^{N}\left\langle f_{j}, H f_{j}\right\rangle
$$

Using Lemma 2.2, this will imply a lower bound of $\operatorname{tr}\left(H \mathbf{1}_{(-\infty, 0)}(H)\right)$.

Consider a partition of unity of $\mathbb{R}$

$$
\left.\psi_{1}^{2}+\psi_{2}^{2}=1, \quad \operatorname{supp} \psi_{1} \subset\right]-\infty, 1\left[, \quad \operatorname{supp} \psi_{2} \subset\left[\frac{1}{2}, \infty[,\right.\right.
$$

such that

$$
\left|\nabla \psi_{1}\right|^{2}+\left|\nabla \psi_{2}\right|^{2} \leq C
$$

and $C>0$ is a universal constant.

Let

$$
\tau(h)=\frac{1}{2} \min (|\tan \alpha|, 1) h^{3 / 8},
$$

where $\alpha=\min \left\{\alpha_{\mathrm{s}_{k}}: k=1,2, \ldots, m\right\}$.

Using the partition of unity in (4.6), we put

$$
t(x)=\operatorname{dist}(x, \partial \Omega), \quad \psi_{1, h}(x)=\psi_{1}\left(\frac{t(x)}{\tau(h)}\right), \quad \psi_{2, h}(x)=\psi_{2}\left(\frac{t(x)}{\tau(h)}\right),
$$

for $x \in \bar{\Omega}$. We introduce the potential

$$
V_{h}=h^{2}\left(\left|\nabla \psi_{1, h}\right|^{2}+\left|\nabla \psi_{2, h}\right|^{2}\right) .
$$

It is possible to prove that (see the proof of eq. (5.10) in [10], p. 243)

$$
\sum_{j=1}^{N}\left\langle f_{j}, H f_{j}\right\rangle \geq \sum_{j=1}^{N}\left\langle f_{j}, \psi_{1, h}\left(H-V_{h}\right) \psi_{1, h} f_{j}\right\rangle-C \frac{h}{\tau(h)}\left(1+\frac{h}{\tau(h)^{2}}\right) .
$$

With our choice of $\tau(h)$, the remainder terms in (4.11) are of the order of $h^{5 / 8}=$ $o\left(h^{1 / 2}\right)$. Thus, we have

$$
\sum_{j=1}^{N}\left\langle f_{j}, H f_{j}\right\rangle \geq \sum_{j=1}^{N}\left\langle f_{j}, \psi_{1, h}\left(H-V_{h}\right) \psi_{1, h} f_{j}\right\rangle+o\left(h^{1 / 2}\right) .
$$


Next, we estimate from below the term on the right side of (4.12). Recall the vertices $\mathrm{s}_{k}, k=1,2, \ldots, m$, of the domain $\Omega$. Recall also that the boundary of the domain $\Omega$ consists of smooth curves $\left(\Gamma_{k}\right)$. For each $k$, define

$$
\Gamma_{k}(h)=\left\{x \in \bar{\Omega}: \operatorname{dist}\left(x, \Gamma_{k}\right) \leq \tau(h) \quad \text { and } \operatorname{dist}\left(x, s_{k}\right) \geq \frac{1}{2} h^{3 / 8}\right\},
$$

where $\tau(h)$ is defined in (4.8). Consider a partition of unity of $\mathbb{R}^{2}$

$$
\sum_{k=1}^{m} g_{k}^{2}+\sum_{k=1}^{m} h_{k}^{2}=1, \quad \sum_{k=1}^{m}\left(\left|\nabla g_{k}\right|^{2}+\left|\nabla h_{k}\right|^{2}\right) \leq \frac{C}{h^{3 / 4}},
$$

where $\operatorname{supp} g_{k} \subset B\left(\mathrm{~s}_{k}, h^{3 / 8}\right), \operatorname{supp} h_{k} \subset \Gamma_{k}(h)$ for all $k$, and $C$ is a universal constant.

Then, we have the decomposition formula

$$
\begin{aligned}
\sum_{j=1}^{N}\left\langle f_{j}, \psi_{1, h}\left(H-V_{h}\right) \psi_{1, h} f_{j}\right\rangle & \\
= & \sum_{j=1}^{N} \sum_{k=1}^{m}\left\langle f_{j}, g_{k} \psi_{1, h}\left(H-V_{1, h}\right) g_{k} \psi_{1, h} f_{j}\right\rangle \\
& \quad+\sum_{j=1}^{N} \sum_{k=1}^{m}\left\langle f_{j}, h_{k} \psi_{1, h}\left(H-V_{1, h}\right) h_{k} \psi_{1, h} f_{j}\right\rangle,
\end{aligned}
$$

where

$$
V_{1, h}=V_{h}+h^{2} \sum_{k=1}^{m}\left|\nabla g_{k}\right|^{2}+h^{2} \sum_{k=1}^{m}\left|\nabla h_{k}\right|^{2}
$$

and

$$
\left|V_{1, h}\right| \leq C h^{5 / 4}
$$

We will show that the first term on the right side of (4.14) is of order $o\left(h^{1 / 2}\right)$. Recall the definition of the energy $\mathcal{E}_{\text {corn }}(\alpha, b, R, \lambda)$ in (2.11). We use (3.12) with $u=\psi_{1, h} f_{j}$ and $\chi_{k, h}=g_{k}$ to bound the term $\left\langle f_{j}, g_{k} \psi_{1, h}\left(H-V_{1, h}\right) g_{k} \psi_{1, h} f_{j}\right\rangle$ from below by a quadratic form defined over the sector $\Omega_{\alpha_{s_{j}}}$. Then we use the variational principle of Lemma 2.2 and the lower bound of $V_{1, h}$ to get

$$
\sum_{j=1}^{N}\left\langle f_{j}, g_{k} \psi_{1, h}\left(H-V_{1, h}\right) g_{k} \psi_{1, h} f_{j}\right\rangle \geq-\left(1-C h^{1 / 4}\right) \mathcal{E}_{\mathrm{corn}}\left(\alpha_{\mathrm{s}_{k}}, \mathbf{B}_{k}, h^{-1 / 8}, \tilde{\lambda}\right) .
$$

Here $\tilde{\lambda}=\lambda b^{-1}-1+C h^{1 / 4} \leq C h^{1 / 4}$. The number $\tilde{\lambda}$ appears naturally after replacing $-V_{1, h}$ by the lower bound $-C h^{5 / 4}$ (see [10], pp. 244-245, for more details). 
Clearly, $\tilde{\lambda}$ can be made smaller than an arbitrary positive number. Thus, we can apply Lemma 2.5 and use (2.14). In this way, we get the following lower bound that holds uniformly with respect to $k$

$$
\sum_{j=1}^{N}\left\langle f_{j}, g_{k} \psi_{1, h}\left(H-V_{1, h}\right) g_{k} \psi_{1, h} f_{j}\right\rangle \geq-C h^{3 / 4}=o\left(h^{1 / 2}\right) .
$$

The second term on the right side of (4.14) is bounded as in the proof of eq. (5.26) in [10], pp. 244-248. The following lower bound holds uniformly in $k, N$ and the orthonormal family $\left\{f_{j}\right\}$ :

$$
\begin{aligned}
& \sum_{j=1}^{N}\left\langle f_{j}, h_{k} \psi_{1, h}\left(H-V_{1, h}\right) h_{k} \psi_{1, h} f_{j}\right\rangle \\
& \quad \geq-\frac{h^{1 / 2}}{2 \pi} \int_{\Gamma_{k}} \int_{\mathbb{R}} B(x)^{3 / 2}\left[\mu_{1}(\xi)-\frac{\lambda}{B(x)}\right]_{-} d \xi d x-C h^{5 / 8}
\end{aligned}
$$

Substitution of (4.15) and (4.16) into (4.14) establishes the lower bound (4.5).

Upper bound. The upper bound will be obtained by constructing a specific density matrix $\gamma$ and computing the energy of $\operatorname{tr}\left(\left(P_{h, \Omega}-\lambda h \mathrm{Id}\right) \gamma\right)$. The calculations follow closely those in Section 5.3 in [10], p. 248.

Recall the sets $\Gamma_{k}(h)$ introduced in (4.13). Let $\tau(h)$ be as defined in (4.8). For each $k$, we cover $\Gamma_{k}(h)$ by disjoint squares

$$
K_{j, k}=\left\{x \in \mathbb{R}^{2}:\left|x-z_{j, k}\right|<\tau\right\}, \quad 1 \leq j \leq N_{k},
$$

where the points $\left(z_{j, k}\right)$ are on $\Gamma_{k}$ and equally spaced. In each $K_{j, k}$, it is possible to apply a transformation $\Phi_{j, k}(x)=(s, t)$ that straightens $\partial \Omega(t=0$ defines the boundary of $\Omega$ and $s$ measures the arc-length along $\partial \Omega$ ).

Recall the eigenvalues $\left(\mu_{p}(\xi)\right)$ of the harmonic oscillator in (2.3). We introduce an orthonormal basis $\left(u_{p}\right)$ of $L^{2}\left(\mathbb{R}_{+}\right)$:

$$
\left\{\begin{array}{l}
\left(-\partial_{t}^{2}+(t-\xi)^{2}\right) u_{p}(t ; \xi)=\mu_{p}(\xi) u_{p}(t ; \xi), \quad t>0 \\
u_{p}^{\prime}(0 ; \xi)=0 \\
\int_{0}^{\infty}\left|u_{p}(t ; \xi)\right|^{2} d t=1
\end{array}\right.
$$

Let $\chi \in C^{\infty}\left(\mathbb{R}^{2}\right)$ be positive, smooth, have values in $[0,1]$, supported in $B(0,2)$ and equal to 1 on $B(0,1)$. Define $\chi_{j, k}(x)$ to be

$$
\chi_{j, k}(x) \stackrel{\text { def }}{=} \chi\left(\frac{x-z_{j, k}}{\tau(h)}\right) .
$$


We denote by $B_{j, k}=\mathbf{B}\left(z_{j, k}\right)$. For each $(j, k)$, there exists a function $\varphi_{j, k}$ such that

$$
\mathbf{A}-\nabla \varphi_{j, k}=\left(-B_{j, k} x_{2}, 0\right)+\mathcal{O}\left(\left|x-z_{j, k}\right|\right) \quad \text { in } K_{j, k}
$$

Define

$$
\tilde{f}_{j, p, k}((s, t) ; h, \xi) \stackrel{\text { def }}{=}\left(\frac{B_{j, k}}{h}\right)^{1 / 4} e^{-i \xi s \sqrt{B_{j, k} / h}} u_{p}\left(\sqrt{\frac{B_{j, k}}{h}} t ; \xi\right) .
$$

We get - by the coordinate transformation $\Phi_{j, k}-$ the function in $\Omega$

$$
f_{j, p, k}(x ; h, \xi)=\left(\frac{1}{2 \pi \sqrt{h / B_{j, k}}}\right)^{1 / 2} \tilde{f}_{j, p, k}\left(\Phi_{j, k}(x) ; h, \xi\right) \chi_{j, k}(x) \psi_{1, h}(x) .
$$

Next, let $K>0$ and define the function

$$
\begin{aligned}
& f_{p}(x ; \xi) \\
& \quad=\sum_{k=1}^{m} \sum_{j=1}^{N_{k}} M_{p}(h, \xi, j, k ; K) e^{i \varphi_{j, k}(x) / h} \chi_{j, k}(x) \psi_{1, h}(x) \tilde{f}_{j, p, k}\left(\Phi_{j, k}(x) ; h, \xi\right),
\end{aligned}
$$

where $M_{p}(h, \xi, j, k ; K)=M^{K}(h, \xi, j, k) \mathbf{1}_{\{p=1\}}$ and $M^{K}(h, \xi, j, k)$ is the characteristic function of the set

$$
\left\{(\xi, j, k): \frac{\lambda}{B_{j, k}}-\mu_{1}(\xi) \geq 0,|\xi| \leq K\right\} .
$$

Let $\Pi_{p}^{\text {bnd }}(h, \xi)$ be the operator with integral kernel in $L^{2}(\Omega)$ whose integral kernel is defined by

$$
\Pi_{p}^{\mathrm{bnd}}(h, \xi)\left(x, x^{\prime}\right)=f_{p}(x ; \xi) \overline{f_{p}\left(x^{\prime} ; \xi\right)}
$$

Define the density matrix

$$
\gamma=\sum_{p=1}^{\infty} \Pi_{p}^{\mathrm{bnd}}(h, \xi)
$$

Clearly $0 \leq \gamma$ as an operator on $L^{2}(\Omega)$. Furthermore, there exists a constant $C>0$ such that

$$
\gamma \leq(1+C \tau(h))
$$

in the quadratic form sense. Details concerning the derivation of (4.22) are give in the proof of eq. (5.34) in [10]. Moreover (see eq. (5.37) in [10]) we have $\operatorname{tr}\left[\left(P_{h, \Omega}-\lambda h\right) \gamma\right] \leq-\frac{h^{1 / 2}}{2 \pi} \int_{-K}^{K} \int_{\partial \Omega} \mathbf{B}(x)^{3 / 2}\left[\frac{\lambda}{\mathbf{B}(x)}-\mu_{1}(\xi)\right]_{+} d s(x) d \xi+C K h^{3 / 4}$ 
Let us mention that, while estimating the term on the left side of (4.23), the discrete sum over $j$ and $k$ becomes a Riemann sum as $h \rightarrow 0$, thereby resulting in an integral on the right side of (4.23). Since $K$ can be chosen arbitrarily large, eq. (4.23) implies the asymptotic upper bound

$\operatorname{tr}\left[\left(P_{h, \Omega}-\lambda h\right) \gamma\right] \leq-\frac{h^{1 / 2}}{2 \pi} \int_{-\infty}^{\infty} \int_{\partial \Omega} \mathbf{B}(x)^{3 / 2}\left[\frac{\lambda}{\mathbf{B}(x)}-\mu_{1}(\xi)\right]_{+} d s(x) d \xi+o\left(h^{1 / 2}\right)$.

\section{References}

[1] J. Avron, I. Herbst, and B. Simon, Schrödinger operators with magnetic fields. I. General interactions. Duke Math. J. 45 (1978), 847-883. MR 518109 Zbl 0399.35029

[2] V. Bonnaillie, On the fundamental state energy for a Schrödinger operator with magnetic field in domains with corners. Asymptot. Anal. 41 (2005), 215-258. MR 2127997 Zbl 1067.35054

[3] V. Bonnaillie-Noël and S. Fournais, Superconductivity in domains with corners. Rev. Math. Phys. 19 (2007), 607-637 MR 2340675 Zbl 1144.82078

[4] C. Bolley and B. Helffer, An application of semi-classical analysis to the asymptotic study of the supercooling field of a superconducting material. Ann. Inst. H. Poincaré Phys. Théor. 58 (1993), 189-233. MR 1217119 Zbl 0779.35104

[5] Y. Colin de Verdière, L'asymptotique de Weyl pour les bouteilles magnétiques. Comm. Math. Phys. 105 (1986), 327-335. MR 0849211 Zbl 0612.35102

[6] H. D. Cornean, S. Fournais, R.L. Frank, and B. Helffer, Sharp trace asymptotics for a class of 2D-magnetic operators. Preprint 2012. arXiv:1108.0777.

[7] M. Dauge and B. Helffer, Eigenvalues variation I. Neumann problem for Sturm-Liouville operators. J. Differential Equations, 104 (1993), 243-262. MR 1231468 Zbl 0784.34021

[8] L. Erdös and J.P Solovej, Semiclassical eigenvalue estimates for the Pauli operator with strong non-homogeneous magnetic fields. II. Leading order asymptotic estimates. Comm. Math. Phys. 188 (1997), 599-656. MR 1473314 Zbl 0909.47052

[9] S. Fournais and B. Helffer, Spectral methods in surface superconductivity. Progress in Nonlinear Differential Equations and Their Applications 77. Birkhäuser, Basel, 1994. MR 2662319 Zbl 1256.35001

[10] S. Fournais and A. Kachmar, On the energy of bound states for magnetic Schrödinger operators. J. Lond. Math. Soc. 80 (2009) 233-255. MR 2520387 Zbl 1179.35203

[11] R. L. Frank, On the asymptotic number of edge states for magnetic Schrödinger operators. Proc. Lond. Math. Soc. (3) 95 (2007), 1-19. MR 2329546 Zbl 1131.35076

[12] P. Grisvard, Elliptic problems in nonsmooth domains. Monographs and Studies in Mathematics 24. Pitman, Boston, MA, 1985. MR 0775683 Zbl 0695.35060

[13] B. Helffer and X. B. Pan, Reduced Landau-de Gennes functional and surface smectic state of liquid crystals. J. Funct. Anal. 255 (2008) 3008-3069. MR 2464569 Zbl 1153.49003

[14] H. Jadallah, The onset of superconductivity in a domain with a corner. J. Math. Phys. 42 (2001), 4101-4121. MR 1852538 Zbl 1063.82041 
[15] B. Helffer and A. Morame, Magnetic bottles in connection with superconductivity. J. Func. Anal. 181 (2001) 604-680. MR 1856278 Zbl 1078.81023

[16] A. Kachmar, On the ground state energy for a magnetic Schrödinger operator and the effect of the de Gennes boundary condition. J. Math. Phys. 47 (2006). Article Id. 072106, 32 pp. MR $2250285 \mathrm{Zbl} 1112.81035$

[17] A. Kachmar, Weyl asymptotics for magnetic Schrödinger operators and de Gennes' boundary condition. Rev. Math. Phys. 20 (2008), 901-932. MR 2450889 Zbl 1167.82024

[18] E. H. Lieb, J. P. Solovej, and J. Yngvason, Asymptotics of heavy atoms in high magnetic fields. II. Semiclassical regions. Comm. Math. Phys. 161 (1994) 77-124. MR 1266071 Zbl 0807.47058

[19] X.-B. Pan, Analogies between superconductors and liquid crystals: nucleation and critical fields. In H. Kozono, T. Ogawa, K. Tanaka, Y. Tsutsumi, and E. Yanagida (eds.), Asymptotic analysis and singularities-elliptic and parabolic PDEs and related problems. Proceedings of the $14^{\text {th }}$ Mathematical Society of Japan (MSJ) International Research Institute on Asymptotic Analysis and Singularity held at Tohoku University, Sendai, July 18-27, 2005. Advanced Studies in Pure Mathematics 47-2. Mathematical Society of Japan, Tokyo, 2007, 479-518. MR 2387252 Zbl 05245138

[20] A. Iwatsuka and H. Tamura, Asymptotic distribution of eigenvalues for Pauli operators with nonconstant magnetic fields. Duke Math. J. 93 (1998), 535-574. MR 1626727 Zbl 0948.35091

Received August 13, 2012; revised November 28, 2012

Ayman Kachmar, Lebanese University, Department of Mathematics, Hadath, Lebanon

Lebanese International University, School of Arts and Sciences, Beirut, Lebanon

E-mail: ayman.kashmar@liu.edu.lb

Abdallah Khochman, Lebanese University, Department of Mathematics, Hadath, Lebanon

E-mail: akhochman@hotmail.fr 